\title{
Study of Weak Periodic Signals in the EEG Signals and Their Relationship with Postsynaptic Potentials
}

\author{
Mahmut AKILLI ${ }^{1}$, Nazmi YILMAZ ${ }^{2}$
}

\begin{abstract}
In this paper, weak periodic signals detected in the EEG signals were analysed for each region of the brain and its relationships with postsynaptic potentials were investigated. For this, EEG signals were collected from 16 different channels according to the international standard channel 10/20 system in the scalp of two patients, one epileptic and one nonepileptic. In our recent work, we have detected weak periodic signals in the EEG signals using Duffing oscillator system. In this paper, we have used a compact method by combining Duffing Oscillator System with scale index which is based on wavelet analysis in order to make the determination of the weak periodic signals more straightforward in practice. A number of weak periodic signals were detected in the range of frequencies $4.7-16 \mathrm{~Hz}$ in all channels, however, these were less observed in epileptic EEG signals than non-epileptic. This methodology illustrates that weak periodic signals can be used to distinguish epileptic EEG signals from non-epileptic EEG signals. We have revealed some findings that the weak periodic signals are the postsynaptic potentials of the brain. Therefore, we can measure some frequencies values of the postsynaptic potentials within the EEG signals with this methodology.
\end{abstract}

Index Terms - EEG signals, the weak periodic signals, Duffing oscillator system, wavelet analysis, scale index, postsynaptic potential

\section{INTRODUCTION}

The Duffing Oscillator System is used for the detection of weak periodic signals embedded in noise and chaotic signals. The method is based on the phase-states of the chaotic oscillator that has the property of sensitive dependence on initial conditions. As a consequence, a weak periodic signal, which is a low amplitude signal, is detected via the state transition from a chaotic state to a periodic state of the driven Duffing oscillator $[1,2,3,4,5,6]$.

In this paper, in order to make this method more successful in detecting weak periodic signals, we combined it with the scale index

Manuscript received May 7, 2018; revised June 20, 2018 and August 6, 2018; accepted August 21, 2018. (Corresponding author: Mahmut Akilli.)

M. Akilli is with the Department of Physics, Institute of Sciences, Istanbul University, 34116 Istanbul, Turkey (e-mail:

akillimahmut@yahoo.com.tr).

N. Yilmaz is with the Department of Physics, College of Sciences, Koç University, 34450 Istanbul, Turkey. method based on wavelet analysis. Because, it is very difficult and time consuming to only scan through the phase state diagrams of Duffing oscillator system when searching for weak periodic signals in a chaotic signal. Scale index parameters, similar to the maximum Lyapunov exponents [7] gives quantitative measurements on the chaotic state of the dynamical systems. Scale index gives a measure of the degree of non-periodicity of the dynamical systems in the range of parameter 0 and 1 . When the calculated scale index parameter from a time series of a dynamical system has a value higher than 0 , the system is considered chaotic. When the system is periodic, the parameter converges to 0 . This condition allows us to calculate the scale index parameters for the EEG signals embedded into the Duffing equation directly without the need to analyze the phase state diagrams. In scale index parameter versus signal frequency graph, the weak periodic signals are detected as the signal components with frequency values corresponding to near zero scale index parameters $[8,9]$.

In our recent work, we have detected weak periodic signals in EEG signals by using Duffing oscillator system [10]. In this work, we have used EEG signals data obtained from 16 channels in the scalp of two patients, one being epileptic and the other being non-epileptic. The weak periodic signals are detected by embedding epileptic and nonepileptic EEG signals respectively into Duffing equation as input signal. Weak periodic signals in the epileptic and non-epileptic EEG signals are observed in the graphs of scale index parameter versus EEG signal frequency. This graphs illustrate that weak periodic signals can be used to differentiate between epileptic EEG signals from non-epileptic EEG signals.

We have searched for the answer to the question "What do the weak periodic signals of the EEG represent?" We put forward a hypothesis that the weak periodic signals are probably the Postsynaptic potentials of the brain. We have explained as that evoked potentials are the result of resonance states of weak periodic signals $[11,12,13$, $14,15,16,17]$.

Data: EEG signals used in this paper were obtained from two patients; the EEG data was approved by Celal Bayar University Medical Faculty Ethics Committee in 2009.

\section{METHODS}

\section{A. The Weak Signal Detection Method Based on Duffing Equation}

The basic form of the Duffing equation can be written as below;

$$
\frac{d^{2} x}{d t^{2}}+\delta \frac{d x}{d t}-x+x^{3}=\gamma \cos (t)
$$


Where $\delta$ the ratio of damping is, $\gamma \cos (t)$ is the periodic driving force and $-x+x^{3}$ is the nonlinear restoring force. If $\delta$ is fixed, as $\gamma$ varies from smaller to larger values, state of the Duffing Oscillator System changes from small periodic motion to chaotic motion, and then to large-scale periodic motion. $\gamma=\gamma_{c}$ is the threshold value of amplitude on changing from chaos to great periodic motion. The state of the Duffing Oscillator System is determined by this value which is called the bifurcation value $\left(\gamma_{c}\right)$. When $\gamma \geq \gamma_{c}$, Duffing Oscillator System is forced to be in large-scale periodic state. When $\gamma<\gamma_{c}$, the oscillator stays in chaotic state. Hence, if $-\delta \frac{d x}{d t}$ and $\gamma \cos (t)$ are counteracting forces in the Duffing equation (1), the Duffing Oscillator jumps to periodic state [1,2].

In detection of weak periodic signals, the Duffing equation is a widely used method to form a chaotic oscillator system where $\gamma \cos (t)$ is the reference signal, $\gamma$ is amplitude of the reference signal, which is determined by the critical chaotic state of the Duffing equation (1) $[1,2,3,10]$.

In the critical chaotic state, the Duffing equation is very sensitive. When an input signal is embedded into the equation (1), it can be written as;

$$
\frac{d^{2} x}{d t^{2}}+\delta \frac{d x}{d t}-x+x^{3}=\gamma \cos (t)+\text { input }
$$

When the Duffing oscillator system is excited, the system jumps from the critical chaotic state to the large-scale periodic state. This means that there are weak periodic signals in the input signal. If the Duffing Oscillator System stays in the critical chaotic state, it means that there is no weak periodic signal present in the input signal. Duffing oscillator system only jumps to periodic state when the selected frequency value of the reference signal matches the frequency of the weak periodic signal. The weak periodic signals may be periodic or quasi-periodic and have smaller amplitudes than the reference signal $[1,2,3,4,10]$.

Reference frequency is then changed in the Duffing equation (2) to detect weak signals with different frequency components [1].

Defining $t=w_{0} \tau$, where $w_{0}$ is the reference frequency.

$$
\begin{gathered}
x(t)=x\left(w_{0} \tau\right)=x_{*}(\tau) \\
\frac{d x(t)}{d t}=\frac{d x\left(w_{0} \tau\right)}{d\left(w_{0} \tau\right)}=\frac{1}{w_{0}} \frac{d x\left(w_{0} \tau\right)}{d(\tau)}=\frac{1}{w_{0}} \frac{d x_{*}(\tau)}{d \tau} \\
\frac{d^{2} x(t)}{d t^{2}}=\frac{d^{2} x\left(w_{0} \tau\right)}{d\left(w_{0} \tau\right)^{2}}=\frac{1}{w_{0}^{2}} \frac{d^{2} x\left(w_{0} \tau\right)}{d \tau^{2}}=\frac{1}{w_{0}^{2}} \frac{d^{2} x_{*}(\tau)}{d \tau^{2}}
\end{gathered}
$$

We obtain the below equation by substituting (3) into (2), and omitting the subscript of $x$ [1];

$$
\frac{1}{w_{0}^{2}} \frac{d^{2} x}{d \tau^{2}}+\frac{\delta}{w_{0}} \frac{d x}{d \tau}-x+x^{3}=\gamma \cos \left(w_{0} \tau\right)+\text { input }
$$

taking $x_{1}=x, \quad x_{2}=\frac{1}{w_{0}} \frac{d x}{d \tau}$;

the equation yields [1];

$$
\begin{gathered}
\frac{d x_{2}}{d \tau}=w_{0}\left[-\delta x_{2}+x_{1}-x_{1}^{3}+\gamma \cos \left(w_{0} \tau\right)+\text { input }\right] \\
\frac{d x_{1}}{d \tau}=w_{0} x_{2}
\end{gathered}
$$

Calculation of the numerical solutions of the Duffing equation (5) with the fourth-order Runge-Kutta algorithm is performed. Then we plotted the phase space diagram of the Duffing oscillator system which is formed by $x(t)$ and it is derivative $x^{\prime}(t)$.

As shown in the previous works, the fourth-order RungeKutta algorithm was used to solve the equation (5) for $\delta=0.5$, to determine the bifurcation value. Bifurcation value $\left(\gamma_{c}\right)$ changes by the step size $\mathrm{h}[1,18]$. As the step size was selected as $\mathrm{h}=0.1$, Duffing Oscillator System remains in large-scale periodic state for $\gamma_{c}$. Here the reference amplitude $(\gamma)$ is fixed to a value fractionally smaller than the bifurcation value $\gamma_{c}$ to bring the Duffing Oscillator to critical chaotic state.

We detected the unknown frequency values of the weak periodic signals within the input signal by frequency scanning by changing the value of $w_{0}$ in the equation (5). If the value of $w_{0}$ matches the frequency of the weak periodic signal, defining $s(\tau)=a \cos \left(w_{0} \tau\right)$ is a weak periodic signal, $a$ is amplitude of weak periodic signal, the total periodic force of the Duffing Oscillator can be written as below;

$A(\tau)=\gamma \cos \left(w_{0} \tau\right)+a \cos \left(w_{0} \tau\right)=(\gamma+a) \cos \left(w_{0} \tau\right)$

Duffing Oscillator System is forced in large-scale periodic state when $\gamma(\tau)=\gamma+a \geq \gamma_{c}$ is the total periodic amplitude [1].

For the parameters $\delta=0.5, w_{0}=0.8$ and the step size $\mathrm{h}=0.1$, the value of amplitude is fixed at $\gamma=0.8255$ before merging the input signal in equation (5), 10000 iterations were used in the numerical solutions of the equation (5), and the phase space diagram of Duffing Oscillator System was plotted in the critical chaotic state (Figure 1a).

The Duffing oscillator is very competent in detecting signals with very low signal-to-noise ratio $[1,3,4,5,19]$. An example of a simulation is shown below for detecting weak periodic signal submerged in noise;

The input signal consisting of weak periodic signals and noise is fed into equation (5), and we obtain equation (7) where the oscillator changes to periodic state (Figure $1 \mathrm{~b}$ and $1 \mathrm{c}$ )

$\begin{aligned} \frac{d^{2} x}{d t^{2}}+0.5 \frac{d x}{d t}-x+x^{3}= & 0.8255 \cos \left(w_{0} \tau\right)+\operatorname{input}(\tau) \\ \text { input }(\tau) & =s(\tau)+y(\tau)\end{aligned}$

$$
\operatorname{input}(\tau)=s(\tau)+y(\tau)
$$

$s(\tau)$ is the weak periodic signal; $s(\tau)=0.0001 \cos \left(w_{0} \tau\right)$,

$y(\tau)$ is the noise, $y(\tau)=\sigma$.rand, where $\sigma$ is $0.005,0.026$ and 0.03 respectively; rand is producing random number. The results show that only when $\sigma \leq 0.026$ the weak signal is identified reliably (Figure 2d). Therefore, we can conclude that the threshold of the signal to noise ratio of the weak signal should be greater than:

$$
S N R=10 \log _{10}\left(0.5 \frac{a^{2}}{\sigma^{2}}\right)=10 \log _{10}\left(0.5 \frac{(0.0001)^{2}}{(0.026)^{2}}\right)=-51.31 \mathrm{db}
$$
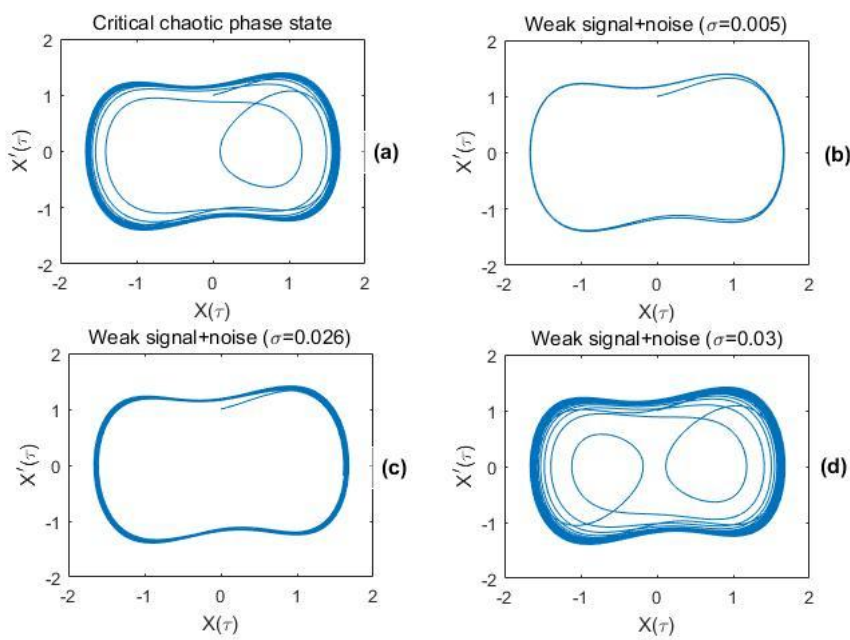

Fig. 1: (a) Chaotic phase state of the Duffing oscillator system before merging the input signal for $\gamma=0.8255$. (b) Phase plane diagram with weak signal and noise $\sigma=0.005$. (c) Phase plane diagram with weak signal and noise $\sigma=0.026$. (d) Phase plane diagram with weak signal and noise $\sigma=0.03$.

\section{B. Combining Duffing Oscillator System with Scale Index}

In order to detect frequency values of the unknown weak periodic signals in the analyzed signal, one needs to keep varying $w_{0}$ value in the equation (5) and observe the phase space of Duffing oscillator in each step to observe a transition from chaotic state to periodic state. This method is impractical and time consuming. We devised a more user friendly and more efficient method in detecting weak periodic signals by the application of the scale index method to support the Duffing oscillator method. The scale index method allows us to scan the signal for wide frequency ranges at once to detect embedded 
weak periodic signal.

Scale index method is a wavelet based method that quantitatively measures a dynamical system's non-periodicity, hence chaotic behavior. In practice, to calculate the scale index for the signal $f$ in the finite time interval $\mathrm{I}=[\mathrm{a}, \mathrm{b}]$, the inner scalogram of $f$ at a scale $s$ is defined by [8]

$$
S^{\text {inner }}(s)=\|W f(u, s)\|_{J(s)}=\left(\int_{c(s)}^{d(s)}|W f(u, s)|^{2} d u\right)^{1 / 2}
$$

Where $J(s)=[c(s), d(s)] \subseteq I$ is the maximal subinterval in $I$. The continuous wavelet transform of function $f$ at time $u$ and scale $s$ :

$$
S(s)=\|W f(u, s)\|=\left(\int_{-\infty}^{+\infty}|W f(u, s)|^{2} d u\right)^{1 / 2}
$$

The inner scalogram is normalized,

$$
\bar{S}^{\text {inner }}(s)=\frac{S^{\text {inner }}(s)}{(d(s)-c(s))^{\frac{1}{2}}}
$$

The scale index of a signal $\mathrm{f}$ is defined in the scale interval $\left[s_{0}, s_{1}\right]$,

$$
i_{\text {scale }}=\frac{\bar{s}^{\text {inner }}\left(s_{\min }\right)}{\bar{S}^{\text {inner }}\left(s_{\max }\right)}
$$

Where $s_{\max }$ is the smallest scale such that $\bar{S}^{\text {inner }}(s) \leq \bar{S}^{\text {inner }}\left(s_{\max }\right)$ for all $s \in\left[s_{0}, s_{1}\right]$, and $s_{\min }$ the smallest scale such that $\bar{S}^{\text {inner }}\left(s_{\text {min }}\right) \leq \bar{S}^{\text {inner }}(s)$ for all $s \in\left[s_{\text {max }}, s_{1}\right]$. (For further details see R.Benitez et al., 2010) [8].

Scale index parameter varies in the range of $0 \leq i_{\text {scale }} \leq 1$. When the scale index parameter converges to 1 , the system strongly is nonperiodic, hence strongly chaotic and when the scale index parameter converges to 0 , the system becomes periodic. In accordance; if Duffing Oscillator System is forced in large-scale periodic state, the scale index parameter converges to 0 , if the Oscillator remains in chaotic state, the Scale Index parameter has a bigger value than $0[8$, 9]. Below, we apply this method to the Duffing equation (8); in the interval of $0.8001 \leq \gamma \leq 0.8300$ and increasing the amplitude $(\gamma)$ by 0.0001 , scale index parameters for the numerical solution of $x(t)$ of the Duffing equation (8) are calculated for each step, we obtain the graph below: (Figure 2). Scale index parameter calculations were performed using Daubechies eight-wavelet function with integer scales between $s_{0}=1$ and $s_{1}=256$.

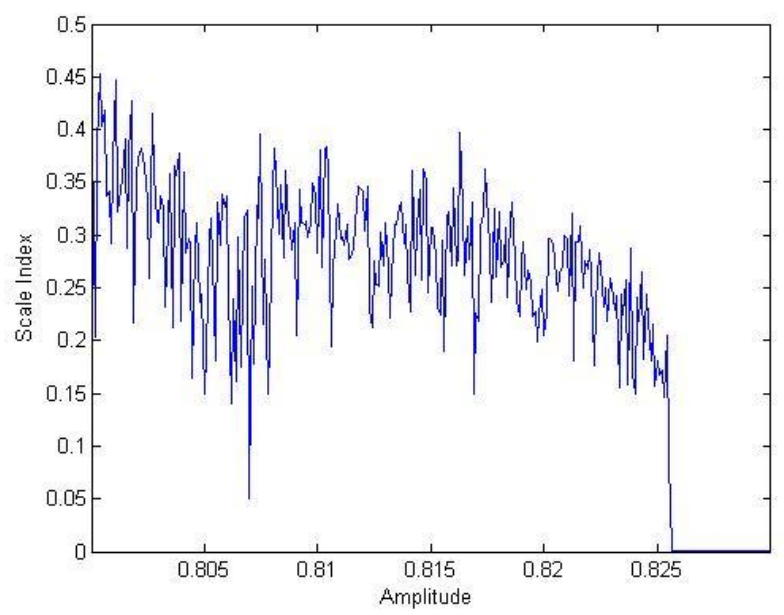

Fig. 2: Scale index parameter versus amplitude graph for the Duffing Oscillator System.

The graph shows that, for $\gamma \geq 0.8256$, Scale Index parameter goes to 0 (periodic state). And for $\gamma<0.8256$ Scale Index parameter is larger than 0 (chaotic state).

Scale index method can be used together with Duffing Oscillator system in detection of weak periodic signals, so that the unknown frequency value of weak periodic signals can be detected by scale index parameters with values nearest to zero without the need to analyze the phase space diagrams. And this unique method of combining Duffing oscillator and scale index will make the weak periodic signal detection much easier. When we perform a simulation to demonstrate this unique method with an input signal; We embed three different weak periodic signals of angular frequency values $0.60,0.70,0.80$ and a noise $y(\tau)=0.02$ rand to the Duffing equation (14). We calculate the scale index parameters for the reference angular frequency interval of $0.50 \leq w_{0} \leq 0.85$ by the step size of 0.01 and obtain the below graph of scale index versus input signal frequency (Figure 3). It can be clearly seen from the graph that scale index parameters nears to 0 for the weak periodic signal angular frequency values indicating periodicity, hence detecting those weak periodic signals within noise.

$\operatorname{input}(\tau)=0.0006 \cos (0.6 \tau)+0.0008 \cos (0.7 \tau)+0.001 \cos (0.8 \tau)+y(\tau)$ (13)

$$
\frac{d^{2} x}{d t^{2}}+0.5 \frac{d x}{d t}-x+x^{3}=0.825 \cos \left(w_{0} \tau\right)+\operatorname{input}(\tau)
$$
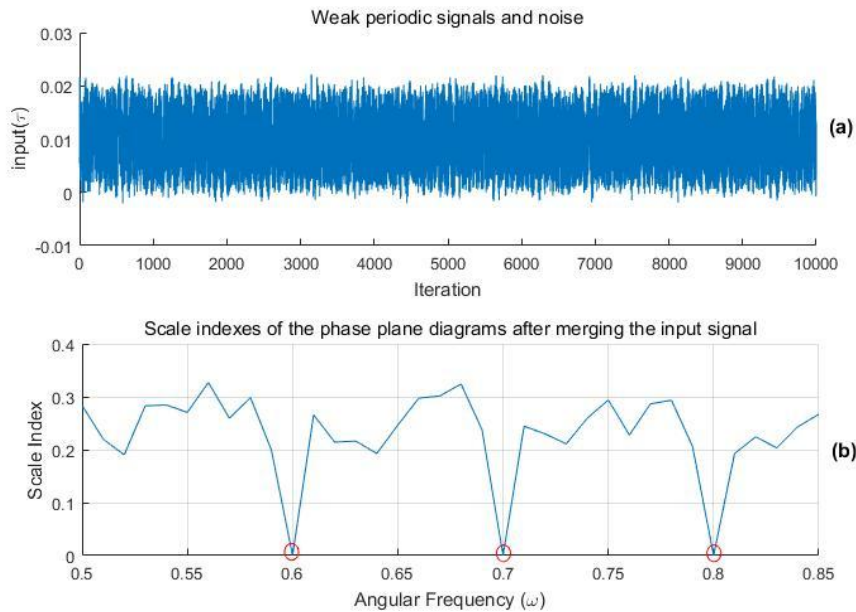

Fig. 3: (a) input( $\boldsymbol{\tau}$ ) signal (b) Scale index versus angular frequency $\left(\boldsymbol{w}_{\mathbf{0}}\right)$ graph for weak periodic signals used as input in the Duffing equation.

\section{RESULTS}

The scale of the input data must fit with the scale of the Duffing Oscillator System. When the scale of the input data is too large compared to the numerical solutions of the Duffing Oscillator, the system collapses and when the scale is too small, the system is not stimulated. Therefore, the input data must be multiplied by $10^{x}$, a gain coefficient, in order to satisfy the compatible scale with the Duffing Oscillator System. The solution to this problem is to scale the input so that it is one digit smaller than $\gamma \cos (t)$ [10].

Before the input signal is embedded in equation (5), Duffing Oscillator System is brought to the critical state as shown in Figure 1a. Then, the EEG signals are embedded into the Duffing equation (5), and the Duffing equation is solved again by fourth order RungeKutta method. To observe weak periodic signals in the EEG signals, frequency scanning must be done with changing $w_{0}$ value in the Duffing equation (5). As a result of scanning, when the frequency of the reference signal $\left(w_{0}\right)$ is equal to one of the frequencies of the weak periodic signals, Duffing Oscillator System in the critical state jumps to the large-scale periodic state as shown in Figure 1b.

For the scanning of the unknown frequency value of weak periodic signals, the scale index method was used with the Duffing Oscillator system, therefore, scale index parameters were calculated for the EEG signals embedded into the Duffing equation (5) as input. Then scale index parameter versus signal frequency graph was plotted. The graph shows that the frequency values of weak periodic signals correspond to scale index parameters converging to " 0 ". 
When $w_{0}$ in the Duffing equation (5) is increased, the bifurcation value $\left(\gamma_{c}\right)$ also increases. Duffing oscillator system is formed in the interval of $0.301 \leq w_{0} \leq 1$. Amplitude values in this interval is chosen as below:

for $0.301 \leq w_{0} \leq 0.350$, amplitude value was chosen as $\gamma=0.8252,\left(\gamma_{c}=0.8253\right)$

for $0.351 \leq w_{0} \leq 0.470$ amplitude value was chosen as

$\gamma=0.8253,\left(\gamma_{c}=0.8254\right)$

for $0.471 \leq w_{0} \leq 0.785$ amplitude value was chosen as $\gamma=0.8254,\left(\gamma_{c}=0.8255\right)$

for $0.786 \leq w_{0} \leq 1 \quad$ amplitude value was chosen as

$\gamma=0.8255,\left(\gamma_{c}=0.8256\right)$;

Therefore, in the angular frequency interval of $0.301 \leq w_{0} \leq 1$, $w_{0}$ value is increased by 0.001 and the scale index parameter is calculated for each step. This angular frequency scanning corresponds to frequency values between $4.791 \mathrm{H} \mathrm{z}$ and $15.916 \mathrm{~Hz}$.

EEG signals used in this paper are obtained from two patients, one being epileptic and the other is non-epileptic [20]. The EEG signals were recorded according to the international standard channel 10/20 system and the sampling rate was $256 \mathrm{~Hz}$. EEG time series were obtained from the first 40 seconds of the recording in 16 channels. The EEG time series are shown in Figures 4 and 5. We investigate the EEG time series by using equation (5).

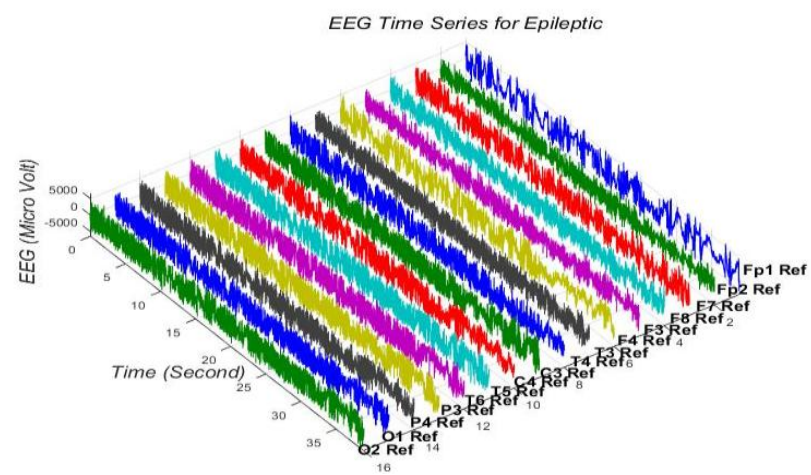

Fig. 4: Epileptic EEG time series were obtained from the first 40 seconds of the recording in 16 channels.

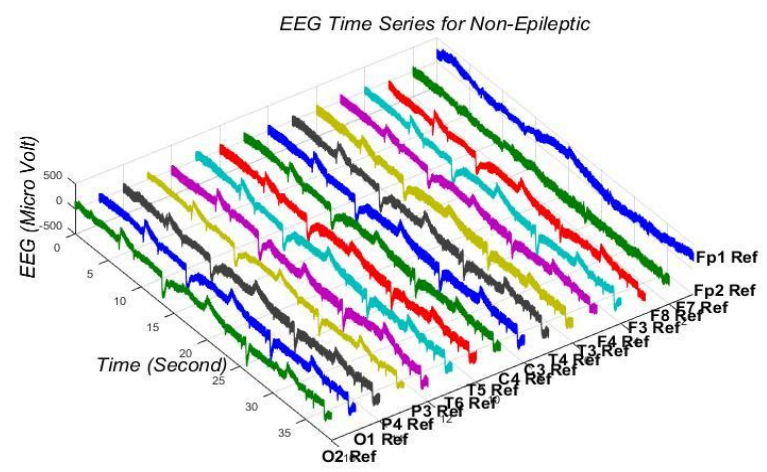

Fig. 5: Non-Epileptic EEG time series were obtained from the first 40 seconds of the recording in 16 channels.

\section{A. Analysis of Epileptic EEG Signals}

a) The Scale Index and the Frequency of Weak Periodic Signals

$\frac{d^{2} x}{d t^{2}}+0.5 \frac{d x}{d t}-x+x^{3}=\gamma \cos \left(w_{0} \tau\right)+10^{\mathrm{x}}\left(\right.$ input $\left._{E E G}\right)$

Epileptic EEG time series were scaled down to compatible range with the Duffing equation (15) by multiplying with a gain coefficient $10^{-5}$ (Figure 6).
The frequency scanning of weak periodic signals was made between the values of $4.791 \mathrm{~Hz}-15.916 \mathrm{~Hz}$. Epileptic EEG time series are put into the Duffing equation (15), then in the interval of $0.301 \leq w_{0} \leq$ 1 and the step size 0.001 , the scale index parameters were calculated.
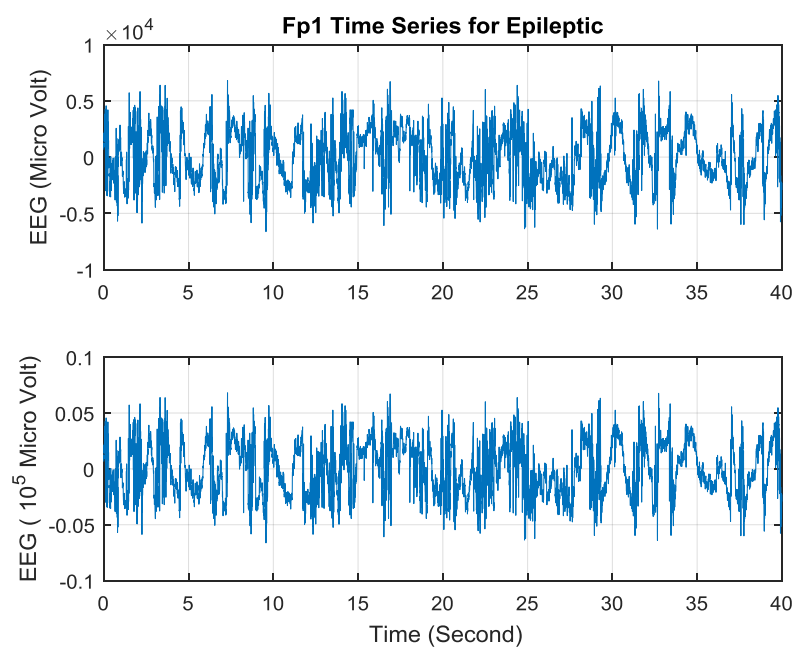

Fig. 6: Epileptic EEG time series were scaled down by multiplying with a gain coefficient $\mathbf{1 0}^{-\mathbf{5}}$

Periodic orbit of the Duffing oscillator is $2 \pi$. In equation (10) for $\delta=0.5, \quad$ according to numerical results, $2 \pi$-periodic orbit corresponds to 100 iteration $[1,2]$. Therefore, frequency and angular frequency relationship can be written as $f=\frac{w_{0}}{2 \pi / 100}$. Using this correlation, scale index parameter versus signal frequency graphs were plotted for all 16-channels. These graphs are shown in figures $7,8,9,10$. These graphs show that scale index parameters that converge to 0 indicate weak periodic signals. The scale index parameter calculations were performed using Daubechies eightwavelet function with integer scales between $s_{0}=1$ and $s_{1}=512$.
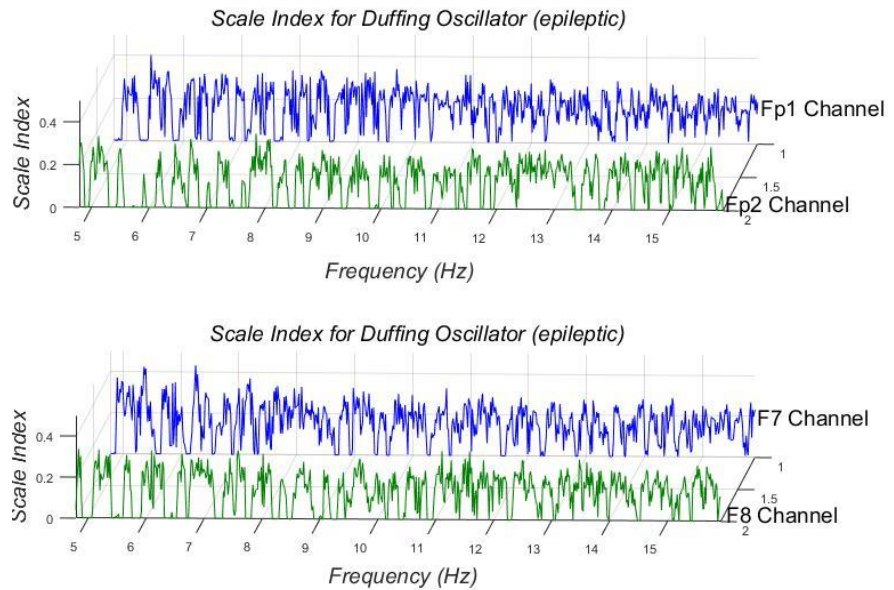

Fig. 7: Scale index versus frequency graphs for the epileptic EEG signals (Fp1, Fp2, F7, F8) used as input in the Duffing equation. 


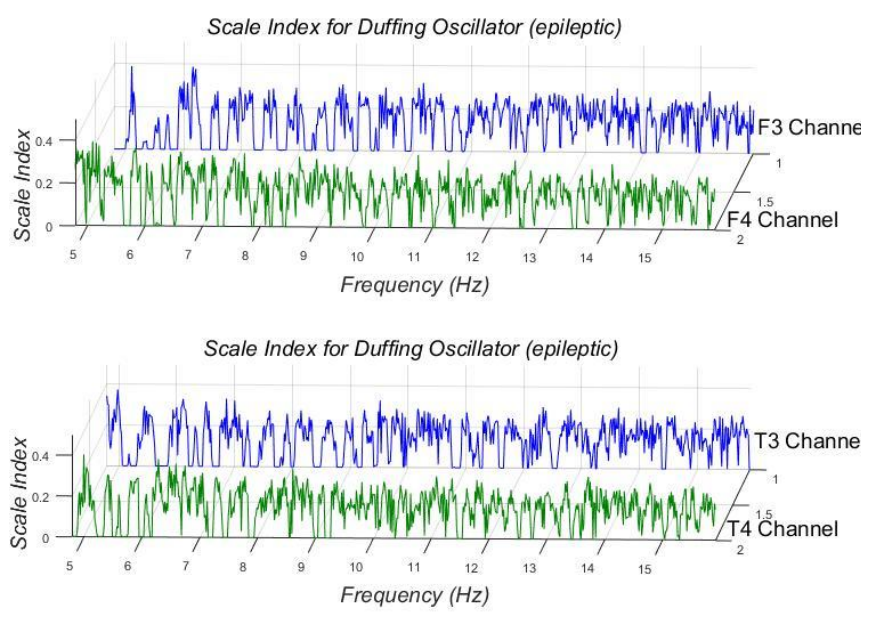

Fig. 8: Scale index versus frequency graphs for epileptic EEG signals (F3, F4, T3, T4) used as input in the Duffing equation.
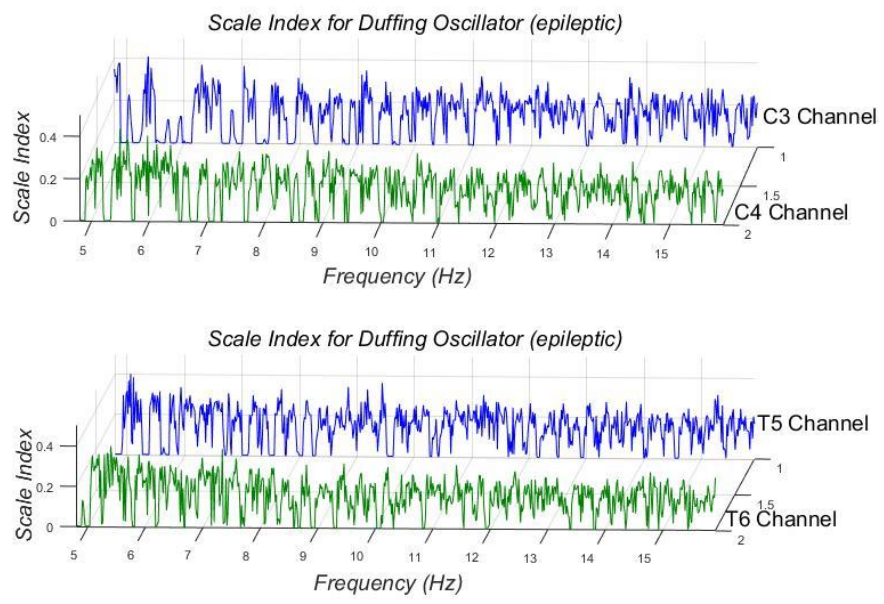

Fig. 9: Scale index versus frequency graphs for epileptic EEG signals (C3, C4, T5, T6) used as input in the Duffing equation.

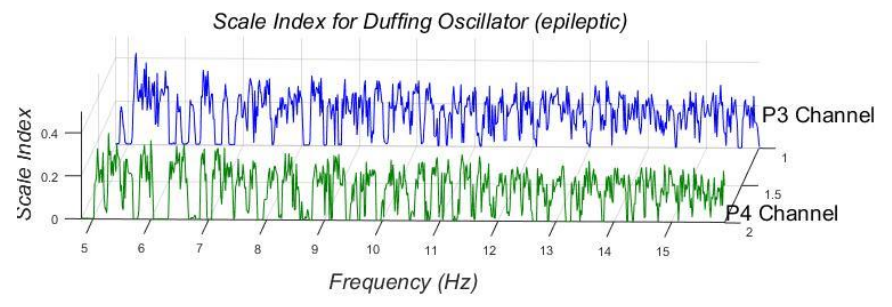

Scale Index for Duffing Oscillator (epileptic)

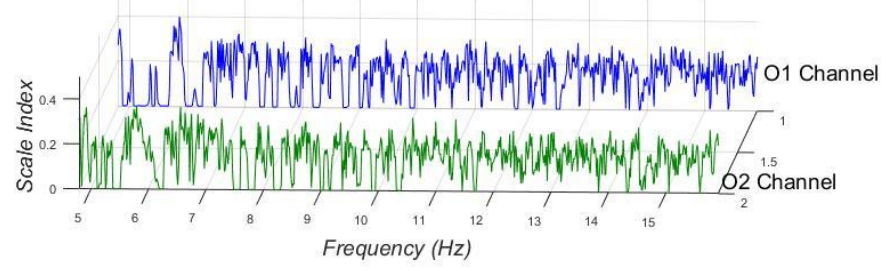

Fig. 10: Scale index versus frequency graphs for epileptic EEG signals (P3, P4, O1, O2) used as input the Duffing equation.

\section{B. Analysis of Non-Epileptic EEG Signals} a) The Scale Index and the Frequency of Weak Periodic
Signals

Non-Epileptic EEG time series were scaled down to compatible range with the Duffing equation (15) by multiplying with a gain coefficient $10^{-4}$ (Figure 11).

In the same manner, scale index - frequency graphs were drawn for non-epileptic EEG time series. This graphics are shown in Figure $12,13,14,15$. Also, weak periodic signal occurrence in EEG signals obtained from epileptic person and non-epileptic person are compared using Fp1 and Fp2 channels in figure 16.
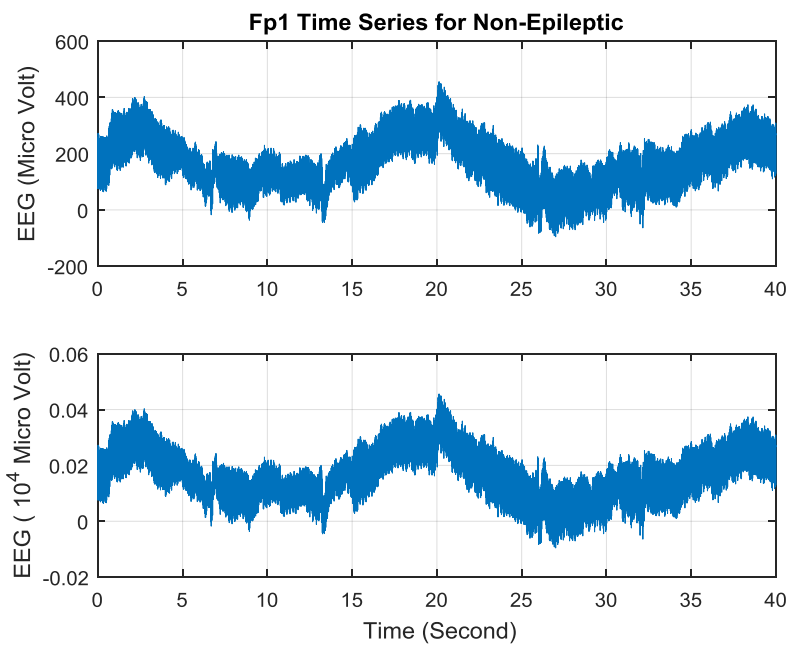

Fig. 11: Non-Epileptic EEG time series were scaled down by multiplying with a gain coefficient $\mathbf{1 0}^{-\mathbf{4}}$
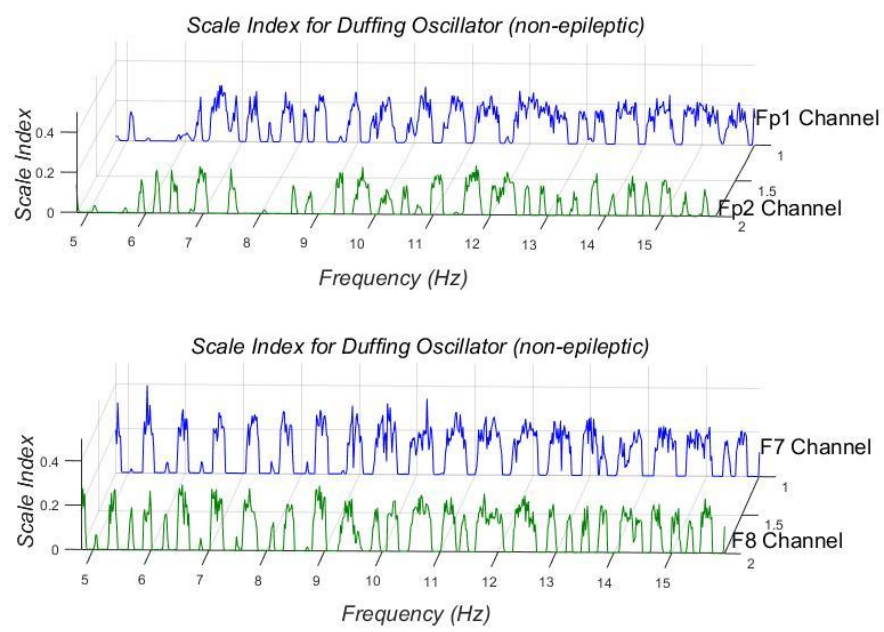

Fig. 12: Scale index versus frequency graphs for non-epileptic EEG signals (Fp1, Fp2, F7, F8) used as input in the Duffing equation. 

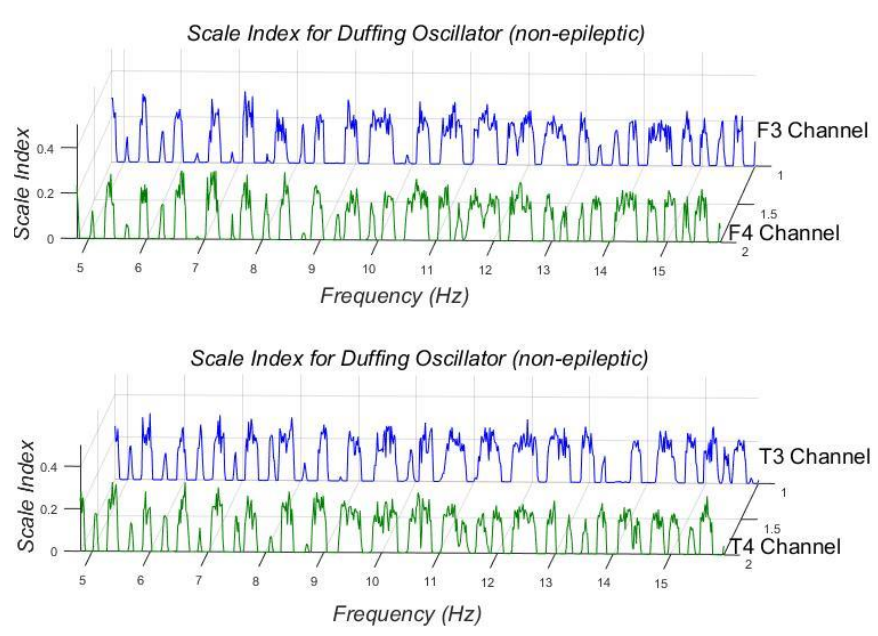

Fig. 13: Scale index versus frequency graphs for non-epileptic EEG signals (F3, F4, T3, T4) used as input in the Duffing equation.

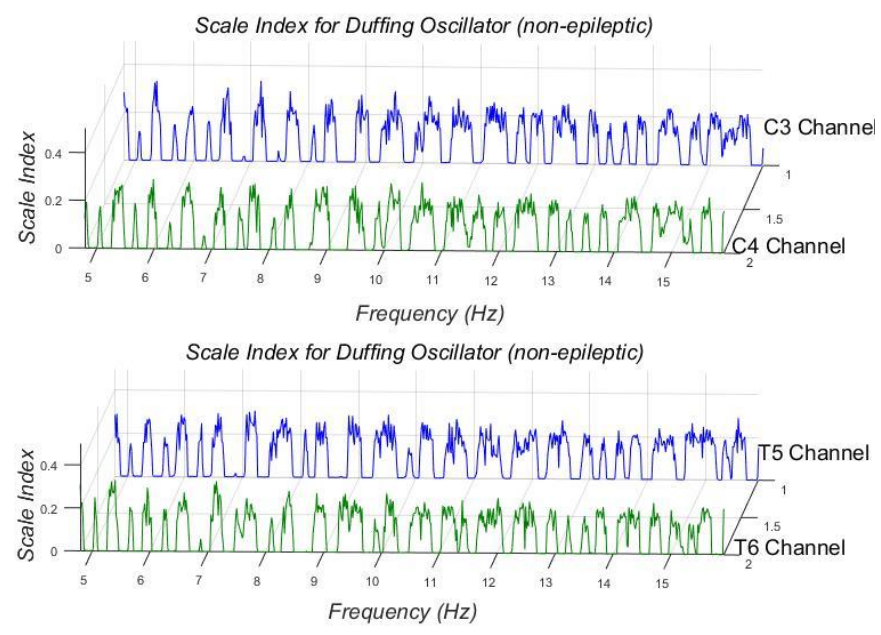

Fig. 14: Scale index versus frequency graphs for non-epileptic EEG signals (C3, C4, T5, T6) used as input in the Duffing equation.
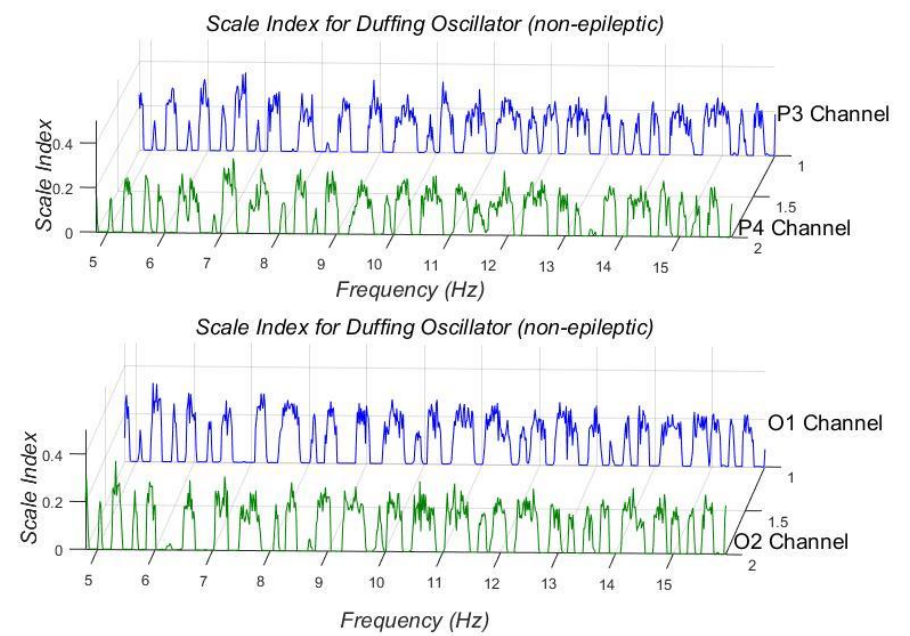

Fig. 15: Scale index versus frequency graphs for non-epileptic EEG signals (P3, P4, O1, O2) used as input in the Duffing equation.

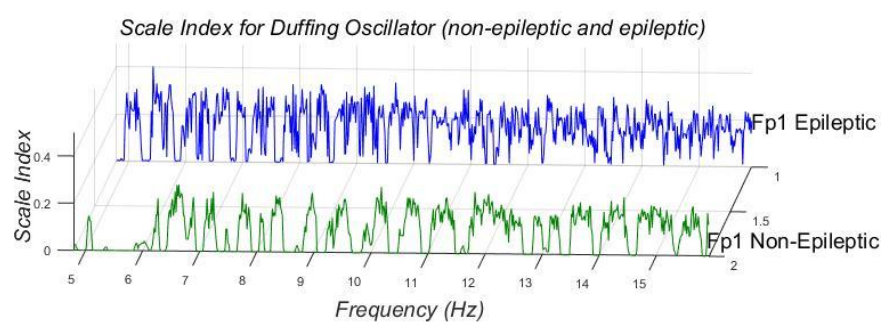

Scale Index for Duffing Oscillator (non-epileptic and epileptic)

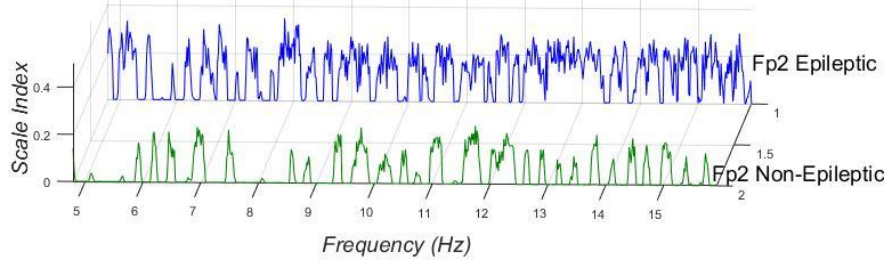

Fig. 16: Comparative scale index graphs were drawn for nonepileptic EEG signals (Fp1, Fp2) and epileptic EEG signals (Fp1, Fp2) used as input in the Duffing equation.

\section{Calculation the Amplitude of Weak Periodic Signals in the EEG Signals}

Amplitude value of weak periodic signals can also be determined using the Duffing Oscillator System. The total periodic amplitude in equation (5) is adjusted [10];

i. If $\gamma+a \geq \gamma_{c}$, Duffing Oscillator System remains in largescale periodic state

ii. If $\gamma+a<\gamma_{c}$, Duffing Oscillator System cannot be transformed into large-scale periodic state, the oscillator remains in chaotic state.

$a$ : Amplitude of weak periodic signal within input $_{E E G}$

To measure amplitude of detected weak periodic signals in the EEG time series, firstly, the angular frequency $\left(w_{0}\right)$ of the reference signal in equation (15) is kept constant to detected any weak periodic signal. Then the amplitude value $(\gamma)$ of the reference signal is adjusted to a point where the large-scale periodic state starts to deteriorate, this amplitude is subtracted from bifurcation value of amplitude $\left(\gamma_{c}\right)$, $\left(a=\gamma_{c}-\gamma\right)$, thus amplitude of weak periodic signal can be obtained. This value is then multiplied with the gain coefficient and the real value of $a$ is found [10]. Table 1 and 2 shows frequency and amplitude values for 4 detected weak periodic signals for each channel.

The lowest amplitude of the detected weak periodic signals and the root mean square of the amplitude of the EEG signals are used in SNR calculation with the assumption that the weak periodic signals are sinusoidal. For Epileptic EEG signal in FP1 channel;

$a=10 \mu \mathrm{V},(\mathrm{Fp} 1)_{\mathrm{rms}}=2310 \mu \mathrm{V}$

$\mathrm{SNR}=10 \log _{10}\left(0.5 \frac{a^{2}}{\mathrm{Fp} 1^{2}}\right)=10 \log _{10}\left(0.5 \frac{(10)^{2}}{(2310)^{2}}\right)=-50.28 \mathrm{db}$

For Non-Epileptic EEG signal in FP1 channel;

$$
\begin{aligned}
& a=1 \mu \mathrm{V},(\mathrm{Fp} 1)_{\mathrm{rms}}=210 \mu \mathrm{V} \\
& \qquad \mathrm{SNR}=10 \log _{10}\left(0.5 \frac{(1)^{2}}{(210)^{2}}\right)=-49.45 \mathrm{db}
\end{aligned}
$$

The both SNR values above are higher than $-51.31 \mathrm{db}$, the threshold SNR value for The Duffing oscillator to be reliable in detecting weak periodic signals, as shown in section $2 \mathrm{~A}$. 
TABLE I

SOME FREQUENCY AND AMPLITUDE VALUES OF WEAK PERIODIC SIGNALS WITHIN THE EPILEPTIC EEG TIME SERIES

\begin{tabular}{|c|c|c|c|c|c|c|c|}
\hline Channel & \begin{tabular}{|l} 
Angular \\
Frequency
\end{tabular} & \begin{tabular}{|l|} 
Frequency \\
$(\mathrm{Hz})$
\end{tabular} & $\begin{array}{l}\text { Amplitude } \\
(\mu \mathrm{V})\end{array}$ & Channel & \begin{tabular}{|l|} 
Angular \\
Frequency
\end{tabular} & $\begin{array}{l}\text { Frequency } \\
(\mathrm{Hz})\end{array}$ & $\begin{array}{l}\text { Amplitude } \\
(\mu \mathrm{V})\end{array}$ \\
\hline \multirow{4}{*}{ Fp1 } & 0.716 & 11.396 & 40 & \multirow{4}{*}{ Fp2 } & 0.993 & 15.804 & 10 \\
\hline & 0.707 & 11.252 & 30 & & 0.867 & 13.799 & 30 \\
\hline & 0.535 & 8.515 & 80 & & 0.749 & 11.921 & 20 \\
\hline & 0.481 & 7.655 & 40 & & 0.59 & 9.390 & 60 \\
\hline \multirow{4}{*}{ F7 } & 0.858 & 13.656 & 30 & \multirow{4}{*}{ F8 } & 0.955 & 15.199 & 10 \\
\hline & 0.806 & 12.828 & 20 & & 0.82 & 13.051 & 20 \\
\hline & 0.77 & 12.255 & 30 & & 0.639 & 10.170 & 20 \\
\hline & 0.603 & 9.597 & 80 & & 0.581 & 9.247 & 70 \\
\hline \multirow{4}{*}{ F3 } & 0.88 & 14.006 & 60 & \multirow{4}{*}{ F4 } & 0.715 & 11.380 & 20 \\
\hline & 0.75 & 11.937 & 40 & & 0.646 & 10.281 & 50 \\
\hline & 0.683 & 10.870 & 50 & & 0.618 & 9.836 & 100 \\
\hline & 0.564 & 8.976 & 70 & & 0.515 & 8.196 & 60 \\
\hline \multirow{4}{*}{ T3 } & 0.908 & 14.451 & 30 & \multirow{4}{*}{ T4 } & 0.843 & 13.417 & 10 \\
\hline & 0.833 & 13.258 & 10 & & 0.786 & 12.510 & 10 \\
\hline & 0.73 & 11.618 & 60 & & 0.652 & 10.377 & 10 \\
\hline & 0.577 & 9.183 & 70 & & 0.578 & 9.199 & 150 \\
\hline \multirow{4}{*}{ C3 } & 0.815 & 12.971 & 90 & \multirow{4}{*}{ C4 } & 0.593 & 9.438 & 10 \\
\hline & 0.648 & 10.313 & 20 & & 0.541 & 8.610 & 30 \\
\hline & 0.522 & 8.308 & 50 & & 0.484 & 7.703 & 40 \\
\hline & 0.491 & 7.815 & 90 & & 0.455 & 7.242 & 40 \\
\hline \multirow{4}{*}{ T5 } & 0.81 & 12.892 & 10 & \multirow{4}{*}{ T6 } & 0.842 & 13.401 & 70 \\
\hline & 0.764 & 12.159 & 10 & & 0.632 & 10.059 & 70 \\
\hline & 0.537 & 8.547 & 50 & & 0.586 & 9.326 & 30 \\
\hline & 0.505 & 8.037 & 60 & & 0.562 & 8.945 & 30 \\
\hline \multirow{4}{*}{ P3 } & 0.978 & 15.565 & 40 & \multirow{4}{*}{ P4 } & 0.869 & 13.831 & 30 \\
\hline & 0.698 & 11.109 & 60 & & 0.705 & 11.220 & 20 \\
\hline & 0.546 & 8.690 & 20 & & 0.626 & 9.963 & 50 \\
\hline & 0.53 & 8.435 & 120 & & 0.55 & 8.754 & 100 \\
\hline \multirow{4}{*}{01} & 0.783 & 12.462 & 100 & \multirow{4}{*}{02} & 0.653 & 10.393 & 60 \\
\hline & 0.738 & 11.746 & 30 & & 0.623 & 9.915 & 60 \\
\hline & 0.621 & 9.884 & 80 & & 0.566 & 9.008 & 20 \\
\hline & 0.567 & 9.024 & 80 & & 0.515 & 8.196 & 100 \\
\hline
\end{tabular}

TABLE II

SOME FREQUENCY AND AMPLITUDE VALUES OF WEAK PERIODIC SIGNALS WITHIN THE NON-EPILEPTIC EEG TIME SERIES

\begin{tabular}{|c|c|c|c|c|c|c|c|}
\hline Channel & \begin{tabular}{|l|} 
Angular \\
Frequency
\end{tabular} & $\begin{array}{l}\text { Frequency } \\
(\mathrm{Hz})\end{array}$ & $\begin{array}{l}\text { Amplitude } \\
(\mu \mathrm{V})\end{array}$ & Channel & \begin{tabular}{|l} 
Angular \\
Frequency
\end{tabular} & $\begin{array}{l}\text { Frequency } \\
(\mathrm{Hz})\end{array}$ & $\begin{array}{l}\text { Amplitude } \\
(\mu \mathrm{V})\end{array}$ \\
\hline \multirow{4}{*}{ Fp1 } & 0.92 & 14.642 & 1 & \multirow{4}{*}{ Fp2 } & 0.996 & 15.852 & 1 \\
\hline & 0.874 & 13.910 & 1 & & 0.879 & 13.990 & 1 \\
\hline & 0.802 & 12.764 & 1 & & 0.749 & 11.921 & 1 \\
\hline & 0.61 & 9.708 & 1 & & 0.653 & 10.393 & 1 \\
\hline \multirow{4}{*}{ F7 } & 0.997 & 15.868 & 2 & \multirow{4}{*}{ F8 } & 0.997 & 15.868 & 1 \\
\hline & 0.874 & 13.910 & 1 & & 0.874 & 13.910 & 1 \\
\hline & 0.765 & 12.175 & 3 & & 0.765 & 12.175 & 2 \\
\hline & 0.61 & 9.708 & 3 & & 0.61 & 9.708 & 2 \\
\hline \multirow{4}{*}{ F3 } & 0.997 & 15.868 & 2 & \multirow{4}{*}{ F4 } & 0.997 & 15.868 & 1 \\
\hline & 0.874 & 13.910 & 1 & & 0.874 & 13.910 & 2 \\
\hline & 0.765 & 12.175 & 2 & & 0.765 & 12.175 & 2 \\
\hline & 0.61 & 9.708 & 2 & & 0.61 & 9.708 & 1 \\
\hline \multirow{4}{*}{ T3 } & 0.997 & 15.868 & 2 & \multirow{4}{*}{ T4 } & 0.997 & 15.868 & 2 \\
\hline & 0.874 & 13.910 & 1 & & 0.874 & 13.910 & 1 \\
\hline & 0.765 & 12.175 & 2 & & 0.765 & 12.175 & 2 \\
\hline & 0.61 & 9.708 & 2 & & 0.61 & 9.708 & 2 \\
\hline \multirow{3}{*}{ C3 } & 0.997 & 15.868 & 1 & \multirow{3}{*}{ C4 } & 0.997 & 15.868 & 2 \\
\hline & 0.765 & 12.175 & 2 & & 0.765 & 12.175 & 3 \\
\hline & 0.61 & 9.708 & 3 & & 0.61 & 9.708 & 3 \\
\hline \multirow{4}{*}{ T5 } & 0.997 & 15.868 & 1 & \multirow{4}{*}{ T6 } & 0.997 & 15.868 & 2 \\
\hline & 0.874 & 13.910 & 1 & & 0.874 & 13.910 & 2 \\
\hline & 0.765 & 12.175 & 2 & & 0.765 & 12.175 & 3 \\
\hline & 0.61 & 9.708 & 2 & & 0.61 & 9.708 & 2 \\
\hline \multirow{4}{*}{ P3 } & 0.997 & 15.868 & 2 & \multirow{4}{*}{ P4 } & 0.997 & 15.868 & 1 \\
\hline & 0.874 & 13.910 & 1 & & 0.874 & 13.910 & 1 \\
\hline & 0.765 & 12.175 & 3 & & 0.765 & 12.175 & 3 \\
\hline & 0.61 & 9.708 & 3 & & 0.61 & 9.708 & 4 \\
\hline \multirow{4}{*}{01} & 0.997 & 15.868 & 2 & \multirow{4}{*}{02} & 0.997 & 15.868 & 2 \\
\hline & 0.874 & 13.910 & 1 & & 0.874 & 13.910 & 2 \\
\hline & 0.765 & 12.175 & 3 & & 0.765 & 12.175 & 3 \\
\hline & 0.61 & 9.708 & 3 & & 0.61 & 9.708 & 3 \\
\hline
\end{tabular}




\section{DISCUSSION}

In this paper, weak periodic signals in the EEG signals were detected by using Duffing Oscillator System with scale index method. When Scale index versus frequency graphs are analyzed, clear differences between the EEG signals of epileptic and nonepileptic patients can be observed. Occurrence frequency of the weak periodic signals in the EEG signals of non-epileptic patient is higher than EEG signals of epileptic patient as shown in Figure 16. Their propagation time is longer, they are synchronized in most signal frequency values as shown in Figures $12,13,14,15$ and they have amplitude values ranging between 1-7 microvolts as shown in Table 2 . In contrast, it can be observed that occurrence frequency of the weak periodic signals in the EEG signals of epileptic patient are considerably low, their propagation time is shorter, they are unsynchronized as shown in Figures 7,8,9,10 and have wider range of amplitude values between 10-240 microvolts as shown in Table 1 .

When we consider those findings, they indicate that weak periodic signals can be used to differentiate epileptic EEG signals from non-epileptic signals. Therefore, this observed difference in weak periodic signals between epileptic and non-epileptic EEG signals can be helpful in epilepsy diagnosis. Predictable and deterministic nature of the weak periodic signals has significant contribution to the analysis and interpretation of EEG signals.

The EEG signals recorded over the scalp are generated by the synaptic potentials of pyramidal neurons. Is there a relation between the weak periodic signals and these postsynaptic potentials?

We think that weak periodic signals are possibly postsynaptic potentials with varying frequencies. Because, postsynaptic potentials, which are subject to spatial and temporal summation, have long and steady propagation time and have distinct waveform. Therefore, some postsynaptic potential with the same frequency values and the propagation time of enough length can be detected by the Duffing oscillator as weak quasi-periodic signals. That is, the postsynaptic potentials of pyramidal neurons activated synchronously can be measured by the Duffing oscillator system. We claim that, the weak periodic signals with given frequency and amplitude values that we detected in this work are likely to be postsynaptic signals. Therefore, we can measure some frequencies values of the postsynaptic potentials within the EEG signals with this methodology.

For we accept that weak periodic signals are Postsynaptic potentials, we think that the brain evoked potentials occur in consequence of the resonance states of the weak periodic signals in certain frequency values which are obtained by direct or peripheral deterministic stimulation (electrical, optical, acoustical, etc). According to our assumption, in EEG signals, the occurrence prevalence of weak periodic signals with broad frequency range indicate that outside stimulus can put those weak periodic signals into resonance state. That is, the amplitude of the resulting weak periodic signals is greatest when the frequency revealed by stimulation is equal to one of the frequencies of the weak periodic signals. Hence, brain evoked potentials are actually the resonance states of the weak periodic signals. Also the weak periodic signals, like brain evoked potentials, are low amplitude signals. The results in this work show that the amplitude values of the detected weak periodic signals are in the same range with brain evoked potentials as shown in Tables 1-2.

\section{REFERENCES}

[1] G. Wang, D. Chen, J. Lin, and X. Chen, "The application of chaotic oscillators to weak signal detection," IEEE Trans. Ind. Electron., vol. 46, pp. 440-444, Apr. 1999.

[2] G Y Wang, S L He. "A quantitative study on detection and estimation of weak signals by using chaotic Duffing oscillators". IEEE T Circuits-I, pp.945-953, 2003.
[3] Li Yue, Yang B J, Zhao X P, et al., "Algorithm of chaotic vibrator to detect weak events in seismic prospecting records", Chinese J Geophys (in Chinese with English abstract), 48(6): 1428-1433, 2005.

[4] Y Li, B Y Yang, Y Yuan, et al, "Ability to detect weak effective seismic signals by utilizing chaotic vibrator system", Chinese Sci Bull, pp.3010-3017, 2006.

[5] Zeng L., Zhang L., "State Identification of Duffing Oscillator Based on Extreme Learning Machine", IEEE Signal Processing Letters, Volume: 25, Issue: 1, Jan. 2018.

[6] Luo WM.,Zhang YR., "Non-periodic pulse signal detection based on variable scale coupled Duffing oscillators", Electronics Letters, Volume: 54, Issue: 5, March 2018.

[7] Wolf, A., Swift, J.B., Swinney, H.L. Vastano, J.A., "Determining Lyapunov Exponents from a Time Series", Physical D, 16: 285-317, 1985.

[8] R. Benitez, V. J. Bolos, M. E. Ramirez, “A Wavelet Based Tool for Studying Non-Periodicity", Computers \& Mathematics with Applications 60(2010) 634-641.

[9] N. Y1lmaz, B. Canbaz, M. Ak1llı, C. Önem, "Study of the stability of the fermionic instanton solutions by the scale index method", Physics Letters A, Volume 382, Issue 32, 2018.

[10] Mahmut AKILLI, "Detecting Weak Periodic Signals in EEG Time Series", Chinese Journal of Physics 54 (2016) 77-85.

[11] Paul L. Nunez and Ramesh Srinivasan, "Electric Fields of the Brain, The Neurophysics of EEG", Oxford University Press. Second Edition 2005, UK.

[12] Basar E. "EEG-Brain dynamics. Relation between EEG and brain evoked potentials". Amsterdam: Elsevier, 1980.

[13] John H. Byrne, "Postsynaptic Potentials and Synaptic Integration", An Introduction to Cellular and Molecular Neuroscience, 2014, Pages 489-507, Academic Press, USA.

[14] Liu F., Wang JF, Wang W., "Frequency sensitivity in weak signal detection", Physical Review E, Vol. 59, Iss. 3 - March 1999.

[15] Yu Y., Liu F.,Wang W., "Frequency sensitivity in HodgkinHuxley systems", Biological Cybernetics, Volume 84, Issue 3, February 2001.

[16] Jung SN, Longtin A, Maler L., "Weak signal amplification and detection by higher-order sensory neurons", Journal of Neurophysiology, Volume 115, Issue 4, April 2016.

[17] Fan H., Wang Y.,Wang H., Lai YC., Wang X., "Autapses promote synchronization in neuronal networks", Scientific Reports, 2018; 8: 580.

[18] J. H. E. Cartwright and O. Piro, "The dynamics of Runge-Kutta methods”, Int. J. Bifurc. Chaos, vol. 2, no. 3, pp. 427-449, Sept. 1992.

[19] A. Jalilvand and H. Fotoohabadi. "The application of Duffing oscillator in weak signal detection." ECTI Transactions on Electrical Eng., Electronics, and Communications 9, no. 1 (2011): 1-6.

[20] T. Zeren, et al., "Nonlinear analysis of EEG signals using Lyapunov exponents in patients with epilepsy", 2nd International Biophysics Congress and Biotechnology at GAP, pp. 7-7, Diyarbakir-Turkey, 2009. 